\title{
Understanding the Core-Halo Relation of Quantum Wave Dark Matter, $\psi$ DM, from 3D Simulations
}

\author{
Hsi-Yu Schive, ${ }^{1}$ Ming-Hsuan Liao, ${ }^{1}$ Tak-Pong Woo, ${ }^{1}$ Shing-Kwong Wong, ${ }^{1}$ \\ Tzihong Chiueh,, , , * Tom Broadhurst, ${ }^{3,4}$ and W-Y. Pauchy Hwang ${ }^{1,2}$ \\ ${ }^{1}$ Department of Physics, National Taiwan University, Taipei 10617, Taiwan \\ ${ }^{2}$ National Center for Theoretical Sciences, National Taiwan University, Taipei 10617, Taiwan \\ ${ }^{3}$ Department of Theoretical Physics, University of the Basque Country UPV/EHU, E-48080 Bilbao, Spain \\ ${ }^{4}$ Ikerbasque, Basque Foundation for Science, E-48011 Bilbao, Spain
}

\begin{abstract}
We examine the nonlinear structure of gravitationally collapsed objects that form in our simulations of wavelike cold dark matter $(\psi \mathrm{DM})$, described by the Schrödinger-Poisson (SP) equation with a particle mass $\sim 10^{-22} \mathrm{eV}$. A distinct gravitationally self-bound solitonic core is found at the center of every halo, with a profile quite different from cores modeled in the warm or self-interacting dark matter scenarios. Furthermore, we show that each solitonic core is surrounded by an extended halo composed of large fluctuating dark matter granules which modulate the halo density on a scale comparable to the diameter of the solitonic core. The scaling symmetry of the SP equation and the uncertainty principle tightly relate the core mass to the halo specific energy, which, in the context of cosmological structure formation, leads to a simple scaling between core mass $\left(M_{c}\right)$ and halo mass $\left(M_{h}\right), M_{c} \propto a^{-1 / 2} M_{h}^{1 / 3}$, where $a$ is the cosmic scale factor. We verify this scaling relation by (i) examining the internal structure of a statistical sample of virialized halos that form in our 3D cosmological simulations, and by (ii) merging multiple solitons to create individual virialized objects. Sufficient simulation resolution is achieved by adaptive mesh refinement and graphic processing units acceleration. From this scaling relation, present dwarf satellite galaxies are predicted to have kpc sized cores and a minimum mass of $\sim 10^{8} M_{\odot}$, capable of solving the small-scale controversies in the cold dark matter model. Moreover, galaxies of $2 \times 10^{12} M_{\odot}$ at $z=8$ should have massive solitonic cores of $\sim 2 \times 10^{9} M_{\odot}$ within $\sim 60$ pc. Such cores can provide a favorable local environment for funneling the gas that leads to the prompt formation of early stellar spheroids and quasars.
\end{abstract}

PACS numbers: 03.75.Lm, 95.35.+d, 98.56.Wm, 98.62.Gq

Accumulating evidences suggest that the Universe contains $\sim 26 \%$ dark matter [1] which interacts primarily through self-gravity. Dark matter comprising very light bosons with a mass $m_{\psi} \sim 10^{-22} \mathrm{eV}$ has been recognized as a viable means of suppressing low mass galaxies and providing cored profiles in dark matter dominated galaxies [2, 3]. Interestingly, this boson mass scale can naturally arise in a non-QCD axion model [4], lending support for the very light boson. The relative deficiency of the observed number of low-mass galaxies is a major problem for standard cold dark matter (CDM) [5] [7, for which a steeply rising mass function is predicted [8]. Furthermore, the dwarf spheroidal galaxies [9 20] and low surface brightness galaxies [21, 22] are generally inferred to have large flat cores of dark matter, at odds with the singular cores required by standard CDM 23, 24]. Complicated baryonic physics such as supernova feedback is required to solve both issues in the CDM paradigm [2534].

Extremely light bosonic dark matter can be assumed to be non-thermally generated and described by a single coherent wave function [2, 35 38], which we term $\psi$ DM. Here solutions to both the missing-satellite and cusp-core problems arise from the uncertainty principle, leading to an effective quantum-mechanical stress tensor that suppresses small-scale structures below a Jeans scale. The
Jeans scale evolves with the cosmic time slowly as $a^{-1 / 4}$, where $a$ is the cosmic scale factor [2, 38], thereby yielding a sharp break in the linear mass power spectrum. This expected behaviour has recently been demonstrated with the first cosmological simulations at sufficiently high resolution, capable of resolving the smallest galaxy halos forming in this context [39].

Warm dark matter (WDM) is also capable of suppressing small-scale linear power by free streaming [40], but it suffers from the Catch 22 problem [41], where the light particle mass required for creating a sufficiently large core $(\sim 1 \mathrm{kpc})$ would prevent the formation of dwarf galaxies in the first place. Collisional CDM does somewhat better in producing cores consistent with observations, but it cannot suppress the number of dwarf galaxies [42, 43]. For these reasons, $\psi \mathrm{DM}$ and scalar-field dark matter composed of extremely light particles have recently begun to attract attention as a viable contender for the long-sought dark matter (e.g., [39, 44, 51]).

Cosmic structures at high redshifts provide stringent tests for all alternative dark matter models attempting to solve the small-scale issues of CDM in the Local Group. For WDM a tension arises when requiring the relatively large cores of dwarf spheroidal galaxies without violating the small scale power constrained by the Lyman- $\alpha$ forest [41, 52 54]. For $\psi \mathrm{DM}$ this problem may be less severe 
due to the sharper small-scale break in its linear power spectrum as compared to WDM [2, 51]. The power spectrum is marginally consistent with the Lyman- $\alpha$ forest observations, while adding a small amount of CDM component $(\sim 10 \%)$ can certainly further relieve the tension 51]. High- $z$ number counts provide another constraint for galaxies at $6 \leq z \leq 8$ [55]. We notice that the $\psi$ DM power spectrum starts to deviate from CDM at $k \sim 7 h \mathrm{Mpc}^{-1}$ [39], corresponding to a halo mass of $\sim 5 \times 10^{9} M_{\odot}$. Above this mass scale the $\psi \mathrm{DM}$ galaxy number density should be close to CDM, and therefore consistent with the observational constraint [55, 56]. Larger $\psi \mathrm{DM}$ simulations with the addition of baryons will be invaluable for supporting these arguments and testing with the forthcoming observations such as JWST [57] and AdvACT [58].

Previous theoretical work on $\psi \mathrm{DM}$ halos mainly focused on two aspects: (i) a stationary soliton profile with or without self-interaction (e.g., [35, 36, 46]), or (ii) a Navarro-Frenk-White (NFW) profile 24] with its inner cusp replaced by a flat core (e.g., 2, 51]). In either case, the detailed connection between cores and halos in the fully nonlinear regime has not been addressed. This question can be best answered by simulations. The first attempt of three-dimensional simulations of the $\psi \mathrm{DM}$ structure formation has come to light only a few years ago [38], revealing complex interference fringes and a halo profile similar to NFW. This work however did not have sufficient spatial resolution for resolving the innermost cores. More recently, Schive et al. [39] made a great leap forward in the $\psi \mathrm{DM}$ simulations by taking advantage of an adaptive mesh refinement (AMR) scheme powered by graphic processing units (GPU) acceleration [59]. A prominent solitonic core is found in every halo, appearing as a self-bound mass clump superposed on the NFW profile (see Fig. 1). This surprising core configuration is apparently different from the linear prediction of $\psi \mathrm{DM}$ [51], WDM [41], and collisional dark matter [43], in all of which a constant-density core is introduced truncating the otherwise cuspy NFW profile. Using the stellar phase-space distribution of the Fornax dwarf spheroidal galaxy, the soliton profile is found to be consistent with observations assuming $m_{\psi}=\left(8.0_{-2.0}^{+1.8}\right) \times 10^{-23} \mathrm{eV}$. Furthermore, this work demonstrates that $\psi \mathrm{DM}$ can clear the Catch 22 problem facing WDM.

In this Letter, we examine the relationship between the solitonic core and the host halo, which we quantify statistically with simulations. We demonstrate that the solitonic core and the halo always coexist in a relaxed, self-bound system of $\psi \mathrm{DM}$. The core mass is tightly related to the halo specific energy, which, for cosmological structure formation, leads to a simple redshift-dependent core-halo mass relation.

Wave mechanics of $\psi \mathrm{DM}$ is governed by the Schrödinger-Poisson (SP) equation [60, 61]. In an expanding universe, the equation can be written in the co- moving coordinates as

$$
\left[i \frac{\partial}{\partial \tau}+\frac{\nabla^{2}}{2}-a V\right] \psi=0
$$

and

$$
\nabla^{2} V=4 \pi\left(|\psi|^{2}-1\right)
$$

where the comoving length $\boldsymbol{x}$ is normalized to $\left(\frac{3}{8 \pi} H_{0}^{2} \Omega_{m 0}\right)^{-1 / 4}\left(m_{\psi} / \hbar\right)^{-1 / 2}$, the time normalized to $d \tau \equiv\left(\frac{3}{8 \pi} H_{0}^{2} \Omega_{m 0}\right)^{1 / 2} a^{-2} d t$, and the wave function $\psi$ normalized to $\left(\rho_{m 0} / m_{\psi}\right)^{1 / 2}$. Here $H_{0}, \Omega_{m 0}$ and $\rho_{m 0}$ are the present Hubble parameter, matter density parameter and background mass density, respectively, and $V$ the gravitational potential. An important feature of the $\mathrm{SP}$ equation is its scaling symmetry [60, 62]. It can be easily seen that when $|\psi|^{2} \gg 1$ and $a=$ const., the SP equation remains unchanged under the transformation $(\tau, \boldsymbol{x}, \psi, V) \rightarrow\left(\lambda^{-2} \tau, \lambda^{-1} \boldsymbol{x}, \lambda^{2} \psi, \lambda^{2} V\right)$ for arbitrary $\lambda$. Having very high densities and forming in a short time compared with the Hubble time, all solitonic cores hence conform to this $\lambda$ scaling to a high accuracy. The relevant physical quantities scale as $\left(x_{c}, \rho_{c}, M_{c}, E_{c}\right) \rightarrow$ $\left(\lambda^{-1} x_{c}, \lambda^{4} \rho_{c}, \lambda M_{c}, \lambda^{3} E_{c}\right)$, where $x_{c}, \rho_{c}, M_{c}$ and $E_{c}$ are the core radius, density, mass and energy, respectively. The soliton density profile can be well fit by [39]

$\rho_{c}(x)=\frac{1.9 a^{-1}\left(m_{\psi} / 10^{-23} \mathrm{eV}\right)^{-2}\left(\mathrm{x}_{\mathrm{c}} / \mathrm{kpc}\right)^{-4}}{\left[1+9.1 \times 10^{-2}\left(x / x_{c}\right)^{2}\right]^{8}} M_{\odot} \mathrm{pc}^{-3}$,

accurate to $2 \%$ in the range $0 \leq x \lesssim 3 x_{c}$. Here we define $x_{c}$ as the radius at which the density drops to one-half its peak value, and $M_{c}$ as the enclosed mass within $x_{c}$. Note that $M\left(x \leq 3 x_{c}\right)$ makes up about $95 \%$ of the total soliton mass, and the half-mass radius is $\sim 1.45 x_{c}$.

To address the core-halo configuration, we conduct three structure formation simulations of different realizations with a spatial resolution up to $60 \mathrm{pc}$ in a 2 Mpc comoving box. These runs begin at the matterradiation equality around $z=3,200$ and end at $z=0$. Note that the small simulation box will affect the statistical properties of halos such as the mass function 63], but should have a small impact on the core-halo relation addressed in this Letter which mainly relies on the virialization of each individual halo and is insensitive to the initial power spectrum. We demonstrate this point by tracing several halos in a $20 \mathrm{Mpc}$ box with the same spatial resolution as in the $2 \mathrm{Mpc}$ simulations. Another simulation with a $40 \mathrm{Mpc}$ box is conducted from $z=3,200$ to $z=8$ for probing the high-redshift galaxies. Our results verify that halos at different redshifts all contain self-similar solitonic cores. Density granules of about the same size as the solitonic core are apparent throughout the halos (see Fig. 2 in Ref. [39] for an illustration): an important feature for the core-halo connection and will be explained later. The soliton profile is redshift-dependent. To see this, note that as long 


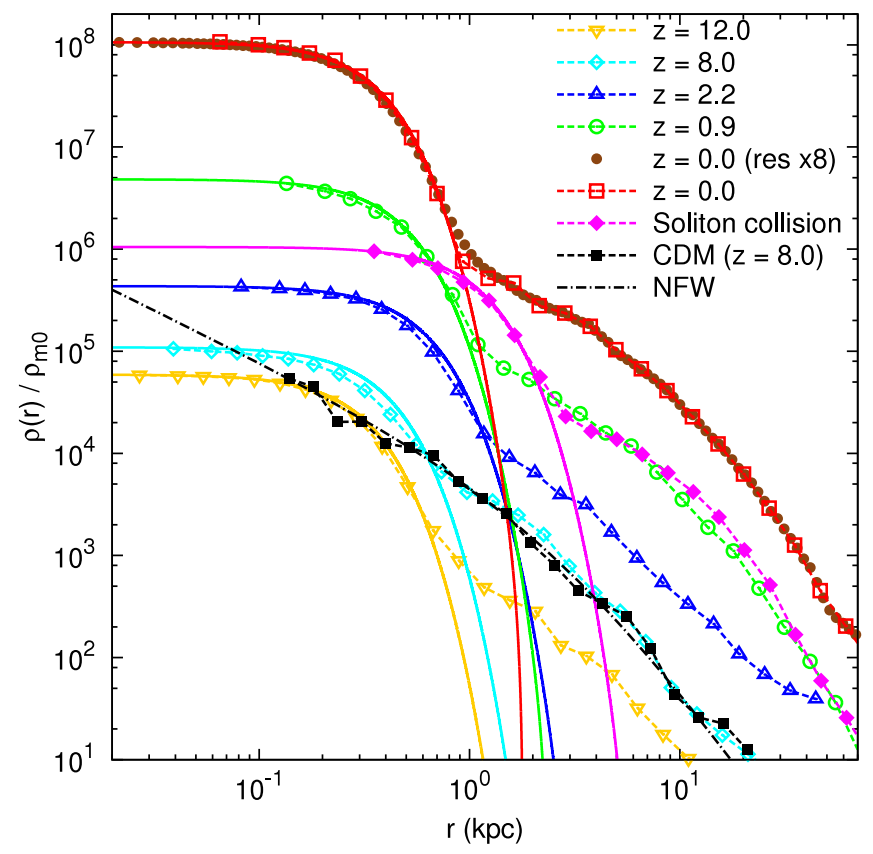

FIG. 1: Density profiles of $\psi \mathrm{DM}$ halos. Dashed lines with various opened symbols show five examples at different redshifts between $12 \geq z \geq 0$. The DM density is normalized to the cosmic background density. A distinct core forms in every halo as a gravitationally self-bound object, satisfying the redshift-dependent soliton solution (solid lines) upon proper $\lambda$ scaling. As a convergence test, filled circles show the same $z=0$ halo (the most massive one) but with eight times higher resolution. Filled diamonds show an example from the soliton collision simulations arbitrarily renormalized to the comoving coordinates at $z=0$. The same $z=8$ halo in a CDM simulation (filled squares) fit by an NFW profile (dot-dashed line) is also shown for comparison.

as a can be regarded as a constant, the SP equation can be rewritten into a redshift-independent form by introducing a set of rescaled variables: $\left(\tau^{\prime}, \boldsymbol{x}^{\prime}, \psi^{\prime}, V^{\prime}\right) \equiv$ $\left(a^{1 / 2} \tau, a^{1 / 4} \boldsymbol{x}, \psi, a^{1 / 2} V\right)$. It follows that the soliton radius in the comoving (unprimed) coordinates scales as $a^{-1 / 4}$ for a fixed peak core density. Figure 1 shows the density profiles of typical halos in the simulations at five different epochs, $z=12.0,8.0,2.2,0.9$ and 0.0 , in the unprimed coordinates. The agreements of the simulation data to both the $\lambda$ and $a$ scalings are excellent.

A question naturally arises concerning the relation between solitonic cores and their host halos. Aided by our structure formation simulations, we find all collapsed objects approximately follow a redshift-dependent core-halo mass relation,

$$
M_{c} \propto a^{-1 / 2} M_{h}^{1 / 3}
$$

The halo virial mass is defined as $M_{h} \equiv$ $\left(4 \pi x_{v i r}^{3} / 3\right) \zeta(z) \rho_{m 0}$, where $x_{v i r}$ is the comoving virial radius and $\zeta(z) \equiv\left(18 \pi^{2}+82\left(\Omega_{m}(z)-1\right)-39\left(\Omega_{m}(z)-\right.\right.$ $\left.1)^{2}\right) / \Omega_{m}(z) \sim 350(180)$ at $z=0(z \geq 1)$ [64]. Note

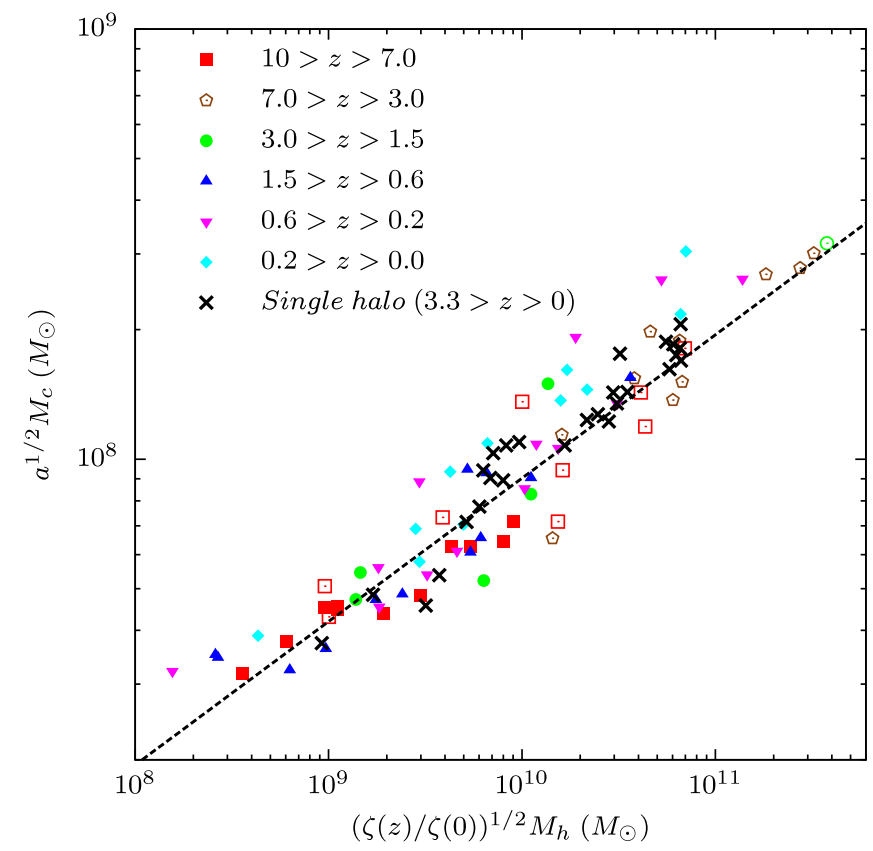

FIG. 2: Core-halo mass relation. Different filled symbols show halos at different epochs in the 2 and $40 \mathrm{Mpc}$ simulations, and open symbols represent the $20 \mathrm{Mpc}$ simulation. Crosses trace the evolution of a single halo. Dashed line shows the analytical prediction given by Eq. (6) (see text for details).

that this definition of virial mass is the same as that for CDM. This is because once an object exceeds the Jeans mass on its way to collapse, the dynamics is almost identical to the cold collapse, for which the Eikonal approximation of wave dynamics to particle dynamics holds until virialization takes place. Figure 2 shows this scaling relation over three orders of magnitude in halo mass from $10^{8}$ to $5 \times 10^{11} M_{\odot}$. We demonstrate the redshift evolution by showing coalescence of the corehalo mass relations of halos at different epochs between $10>z>0$ as well as the evolutionary trajectory of a single halo. Note that low-redshift, massive halos in the $2 \mathrm{Mpc}$ runs show a relatively larger scatter, which could be due to the small box effect, while massive halos in the $20 \mathrm{Mpc}$ run do converge to our analytical prediction. In all cases the deviation of the core mass from Eq. (4) is less than a factor of two. Also note that the halos in the simulations with a mass several times $10^{8} M_{\odot}$ are found to be dominated by the central solitons, a key for estimating the minimum halo mass as will be discussed later.

To understand this core-halo mass relation, we further conduct a set of controlled numerical experiments, where multiple solitons are initially placed randomly with zero velocity and start to merge until the systems relax. Solitons are chosen as a convenient initial condition for their stability. Here we assume $a=$ const. and zero background density. We would like to know whether the core- 

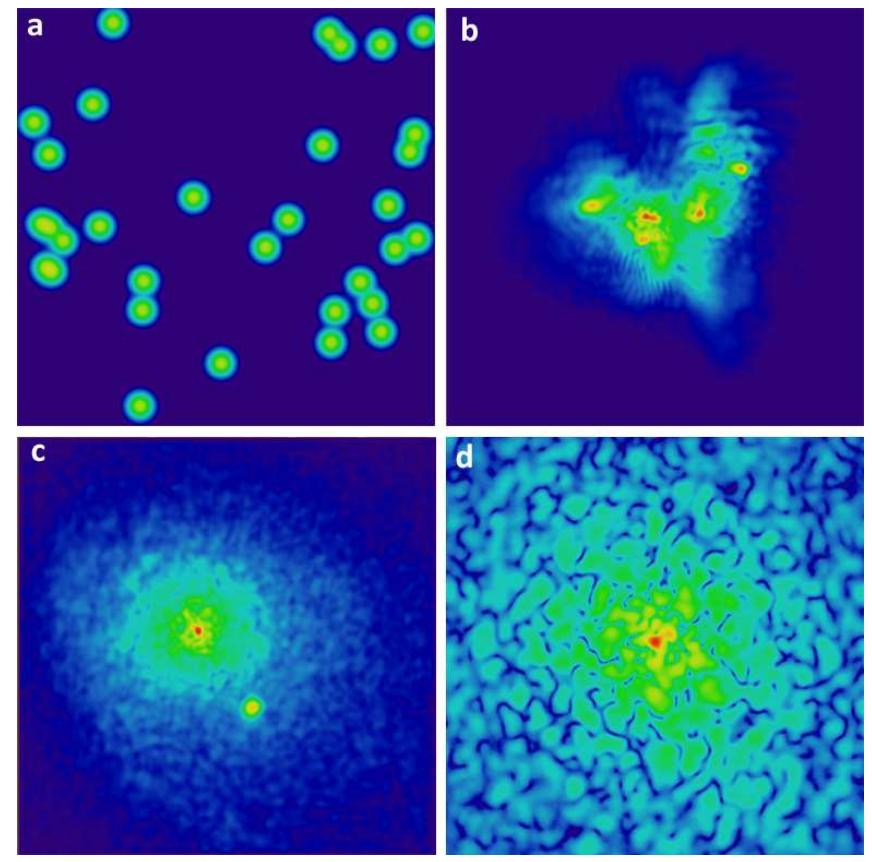

FIG. 3: Snapshots of a soliton collision simulation. Panels (a)-(c) show the projected density distribution at the initial and intermediate stages, and panel (d) shows a close-up of the conspicuous solitonic core at the final stage. Fluctuating density granules resulting from the quantum wave interference appear everywhere and have a size similar to the central soliton.

halo configuration still persists in a different setting from cosmological structure formation, and if so, we want to ascertain what factors determine the soliton scale among the infinite number of self-similar solutions. Intuitively, one expects that the final relaxed state should lose the memory of its initial configuration and thus depends only on the globally conserved quantities, namely, the total mass $M$ and energy $E$ (assuming there is no net angular momentum). We conduct 29 runs in total with different initial conditions of various $M$ and $E$. For the same $M$ and $E$, we repeat runs with different realizations, including different initial soliton numbers ranging from 4 to 128, different soliton sizes and initial positions. Figure 3 shows one example of the soliton collision simulations. The AMR scheme is again adopted in order to achieve sufficient resolution everywhere; in particular, we ensure that every soliton is well resolved with at least $\sim 10^{4}$ cells and verify that $M$ and $E$ remain conserved with at most a few percent error in all simulations.

The resulting relaxed structures that form in these soliton collision experiments are always found to consist of a halo and a solitonic core (see Fig. 1 and panel (d) of Fig. 3), similar to the results of cosmological simulations. The core profiles satisfy the $\lambda$ scaling and the halo profiles are close to NFW. This result establishes that the core-halo configuration is a generic structure of $\psi \mathrm{DM}$ in virialized gravitational equilibrium.

More importantly, as shown in Fig. 4 the core mass follows the relation

$$
M_{c}^{\prime}=\alpha\left(\left|E^{\prime}\right| / M^{\prime}\right)^{1 / 2} .
$$

Here the total kinetic energy, potential energy and mass are defined in the primed (redshift-independent) coordinates as $E_{k}^{\prime} \equiv \frac{1}{2} \int\left|\nabla^{\prime} \psi^{\prime}\right|^{2} d^{3} x^{\prime}, E_{p}^{\prime} \equiv \frac{1}{2} \int\left|\psi^{\prime}\right|^{2} V^{\prime} d^{3} x^{\prime}$, $M^{\prime} \equiv \int\left|\psi^{\prime}\right|^{2} d^{3} x^{\prime}$, and $\alpha$ is a dimensionless constant close to unity. The physical foundation of this relation can be appreciated as follows. The RHS represents the halo velocity dispersion, $\sigma_{h}^{\prime}$, and on the LHS the $\lambda$ scaling demands that $M_{c}^{\prime} \sim x_{c}^{\prime-1}$, the inverse soliton size. Accordingly, Eq. (5) relates the soliton size to the halo velocity dispersion through the uncertainty principle, where $x_{c}^{\prime} \sigma_{h}^{\prime} \sim 1$. This result is non-trivial in that the uncertainty principle is originally a local relation, but here it is found to hold non-locally, relating a core (local) property to a halo (global) property. The non-local uncertainty principle reveals itself in panel (d) of Fig. 3. The inverse halo velocity dispersion is manifested by the size of halo density granules, and the fact that the halo granule size is close to the soliton size provides another perspective to view the finding of Eq. (5). Eigenmode decomposition of the core-halo system can help our understanding of the detailed physics underlying this quantum "thermalization", and it will be presented in a separate work (Wong et al., in preparation).

We are now in a position to understand the physical meaning of the empirical Eq. (4). In the structure formation simulations, we verify that halos at different redshifts all conform to Eq. (5) by taking $E^{\prime}$ and $M^{\prime}$ as the rescaled halo energy $\left(E_{h}^{\prime}\right)$ and virial mass $\left(M_{h}^{\prime}\right)$. Adopting the virial condition in the spherical collapse model $\left|E_{h}^{\prime}\right|=\left|E_{p}^{\prime}\right| / 2 \sim 3 M_{h}^{\prime 2} / 10 x_{v i r}^{\prime}$ and retrieving the redshift dependence then give $M_{c}=\alpha\left(3 M_{h} / 10 x_{v i r}\right)^{1 / 2} a^{-1 / 2}$. Finally, solving $x_{v i r}$ as a function of $M_{h}$ using the definition of virial mass given immediately after Eq. (4) yields the expected core-halo mass relation

$$
M_{c}=\frac{1}{4} a^{-1 / 2}\left(\frac{\zeta(z)}{\zeta(0)}\right)^{1 / 6}\left(\frac{M_{h}}{M_{\min , 0}}\right)^{1 / 3} M_{\min , 0},
$$

where $M_{m i n, 0}=375^{-1 / 4} 32 \pi \zeta(0)^{1 / 4} \rho_{m 0}\left(H_{0} m_{\psi} / \hbar\right)^{-3 / 2} \Omega_{m 0}^{-3 / 4}$ $\sim 4.4 \times 10^{7} m_{22}^{-3 / 2} M_{\odot}$. Here $m_{22} \equiv m_{\psi} / 10^{-22} \mathrm{eV}$ and we have taken $\alpha=1$ and typical values for the cosmological parameters. Eq. (6) is consistent with Eq. (4) apart from an additional slowly varying factor $\zeta(z)^{1 / 6}$. The physical core radius, $r_{c}=a x_{c}$, is inversely proportional to $M_{c}$ and can be expressed as

$$
r_{c}=1.6 m_{22}^{-1} a^{1 / 2}\left(\frac{\zeta(z)}{\zeta(0)}\right)^{-1 / 6}\left(\frac{M_{h}}{10^{9} M_{\odot}}\right)^{-1 / 3} \mathrm{kpc} .
$$

The smallest halo should be close to a single isolated soliton, with a wide core and a steeper outer gradient. 


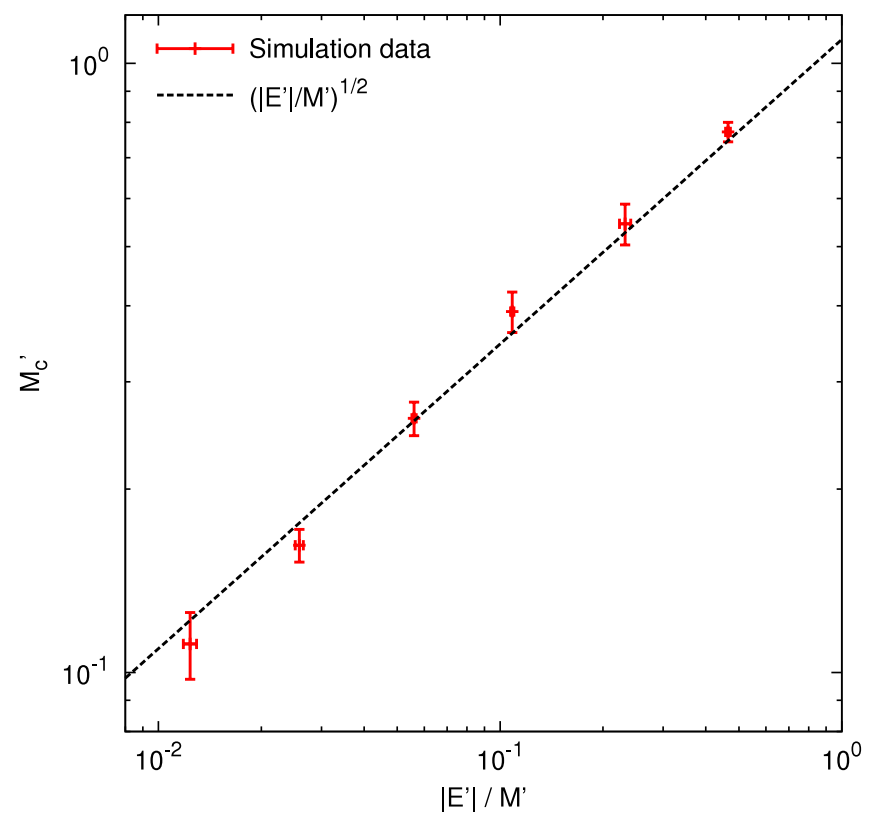

FIG. 4: Scaling relation between core mass and system specific energy in the soliton collision experiments. Error bars represent the root-mean-square scatter of different realizations at a given specific energy bin as well as the fluctuation in different snapshots of each run. Note that the redshift dependence has been absorbed into the rescaled mass $M^{\prime}$ and energy $E^{\prime}$ (see text for details)

Our definition of core mass, $M\left(r \leq r_{c}\right)$, makes up about $25 \%$ of the total soliton mass. Thus by taking $M_{c}=$ $M_{h} / 4$ in Eq. (6) we readily obtain a minimum halo mass $M_{\min }(z)=a^{-3 / 4}(\zeta(z) / \zeta(0))^{1 / 4} M_{\min , 0} \sim 3 \times 10^{8} M_{\odot}$ at $z=8$ for $m_{22}=0.8$, consistent with Fig. 2 and the theoretical prediction [56].

Finally, we conclude this Letter by a conjecture regarding the possible consequences of the early formation of the dense solitonic cores. A present-day galaxy with a typical halo mass of $2 \times 10^{12} M_{\odot}$ will have $M_{c} \sim 5 \times 10^{8} M_{\odot}$ and $r_{c} \sim 160$ pc. For a high-redshift galaxy with the same halo mass, its core mass and gravitational acceleration near the core, $M_{c} / r_{c}^{2}$, will be enhanced by a factor of $a^{-1 / 2}$ and $a^{-3 / 2}$, respectively. This much greater gravitational force may quickly attract a large amount of gas into a small central region, thereby creating an ultra-dense gas favorable for major starbursts and formation of supermassive black holes. For example, a galaxy of $2 \times 10^{12} M_{\odot}$ forming at $z=8$ has a core mass $\sim 2 \times 10^{9} M_{\odot}$ in $\sim 60 \mathrm{pc}$ radius and it captures at least $4 \times 10^{8} M_{\odot}$ gas if the baryon fraction at the core is the same as or above the cosmic mean. If furthermore the gas temperature maintains near the Lyman- $\alpha$ onset temperature, $10 \mathrm{eV}$, this radius is only a factor of two greater than the $30 \mathrm{pc}$ thermal Jeans length of the gas. Such a solitonic core can certainly help the prompt formation of quasars appearing as early as $z=7[65]$.
This work is supported in part by the National Science Council of Taiwan under the grants NSC100-2112-M-002018-MY3 and NSC99-2112-M-002-009-MY3.

* Electronic address: chiuehth@phys.ntu.edu.tw

[1] Planck Collaboration, P. A. R. Ade, N. Aghanim, M. I. R. Alves, C. Armitage-Caplan, M. Arnaud, M. Ashdown, F. Atrio-Barandela, J. Aumont, H. Aussel, et al., A\&A 571, A1 (2014).

[2] W. Hu, R. Barkana, and A. Gruzinov, Phys. Rev. Lett. 85, 1158 (2000).

[3] P. J. E. Peebles, Astrophys. J. 534, L127 (2000).

[4] T. Chiueh, ArXiv e-prints (2014), 1409.0380.

[5] G. Kauffmann, S. D. M. White, and B. Guiderdoni, Mon. Not. R. Astron. Soc. 264, 201 (1993).

[6] A. Klypin, A. V. Kravtsov, O. Valenzuela, and F. Prada, Astrophys. J. 522, 82 (1999).

[7] B. Moore, S. Ghigna, F. Governato, G. Lake, T. Quinn, J. Stadel, and P. Tozzi, Astrophys. J. 524, L19 (1999).

[8] J. Diemand, M. Kuhlen, and P. Madau, Astrophys. J. 657, 262 (2007).

[9] B. Moore, Nature 370, 629 (1994).

[10] R. A. Flores and J. R. Primack, Astrophys. J. Lett. 427, L1 (1994).

[11] J. T. Kleyna, M. I. Wilkinson, G. Gilmore, and N. W. Evans, Astrophys. J. 588, L21 (2003).

[12] T. Goerdt, B. Moore, J. I. Read, J. Stadel, and M. Zemp, Mon. Not. R. Astron. Soc. 368, 1073 (2006).

[13] G. Gilmore, M. I. Wilkinson, R. F. G. Wyse, J. T. Kleyna, A. Koch, N. W. Evans, and E. K. Grebel, Astrophys. J. 663, 948 (2007).

[14] G. Battaglia, A. Helmi, E. Tolstoy, M. Irwin, V. Hill, and P. Jablonka, Astrophys. J. Lett. 681, L13 (2008).

[15] M. G. Walker and J. Peñarrubia, Astrophys. J. 742, 20 (2011).

[16] A. Agnello and N. W. Evans, Astrophys. J. Lett. 754, L39 (2012).

[17] D. R. Cole, W. Dehnen, J. I. Read, and M. I. Wilkinson, Mon. Not. R. Astron. Soc. 426, 601 (2012).

[18] J. R. Jardel and K. Gebhardt, Astrophys. J. 746, 89 (2012).

[19] J. Peñarrubia, A. Pontzen, M. G. Walker, and S. E. Koposov, Astrophys. J. Lett. 759, L42 (2012).

[20] N. C. Amorisco, A. Agnello, and N. W. Evans, Mon. Not. R. Astron. Soc. 429, L89 (2013).

[21] W. J. G. de Blok, S. S. McGaugh, A. Bosma, and V. C. Rubin, Astrophys. J. Lett. 552, L23 (2001).

[22] W. J. G. de Blok and A. Bosma, Astron. \& Astrophys. 385, 816 (2002).

[23] J. Dubinski and R. G. Carlberg, Astrophys. J. 378, 496 (1991).

[24] J. F. Navarro, C. S. Frenk, and S. D. M. White, Astrophys. J. 462, 563 (1996).

[25] J. F. Navarro, V. R. Eke, and C. S. Frenk, Mon. Not. R. Astron. Soc. 283, L72 (1996).

[26] J. I. Read and G. Gilmore, Mon. Not. R. Astron. Soc. 356, 107 (2005).

[27] J. I. Read, M. I. Wilkinson, N. W. Evans, G. Gilmore, and J. T. Kleyna, Mon. Not. R. Astron. Soc. 367, 387 (2006). 
[28] J. Peñarrubia, A. J. Benson, M. G. Walker, G. Gilmore, A. W. McConnachie, and L. Mayer, Mon. Not. R. Astron. Soc. 406, 1290 (2010).

[29] A. Zolotov, A. M. Brooks, B. Willman, F. Governato, A. Pontzen, C. Christensen, A. Dekel, T. Quinn, S. Shen, and J. Wadsley, Astrophys. J. 761, 71 (2012).

[30] A. Pontzen and F. Governato, Mon. Not. R. Astron. Soc. 421, 3464 (2012).

[31] R. Teyssier, A. Pontzen, Y. Dubois, and J. I. Read, Mon. Not. R. Astron. Soc. 429, 3068 (2013).

[32] A. M. Brooks and A. Zolotov, Astrophys. J. 786, 87 (2014).

[33] A. Pontzen and F. Governato, Nature 370, 629 (2014).

[34] G. Kauffmann, Mon. Not. R. Astron. Soc. 441, 2717 (2014).

[35] J. Goodman, New Astron. 5, 103 (2000).

[36] C. G. Böhmer and T. Harko, J. Cosmol. Astropart. Phys. 6, 25 (2007).

[37] P. Sikivie and Q. Yang, Phys. Rev. Lett. 103, 111301 (2009).

[38] T.-P. Woo and T. Chiueh, Astrophys. J. 697, 850 (2009).

[39] H.-Y. Schive, T. Chiueh, and T. Broadhurst, Nature Phys. 10, 496 (2014).

[40] P. Bode, J. P. Ostriker, and N. Turok, Astrophys. J. 556, 93 (2001).

[41] A. V. Macciò, S. Paduroiu, D. Anderhalden, A. Schneider, and B. Moore, Mon. Not. R. Astron. Soc. 424, 1105 (2012).

[42] D. N. Spergel and P. J. Steinhardt, Phys. Rev. Lett. 84, 3760 (2000).

[43] M. Rocha, A. H. G. Peter, J. S. Bullock, M. Kaplinghat, S. Garrison-Kimmel, J. Oñorbe, and L. A. Moustakas, Mon. Not. R. Astron. Soc. 430, 81 (2013).

[44] A. Suárez and T. Matos, Mon. Not. R. Astron. Soc. 416, 87 (2011).

[45] P.-H. Chavanis, Phys. Rev. D 84, 043531 (2011).

[46] V. Lora, J. Magaña, A. Bernal, F. J. Sánchez-Salcedo, and E. K. Grebel, J. Cosmol. Astropart. Phys. 2, 11 (2012).

[47] V. H. Robles and T. Matos, Mon. Not. R. Astron. Soc. 422, 282 (2012).

[48] T. Rindler-Daller and P. R. Shapiro, Mod. Phys. Lett. A
29, 30002 (2014).

[49] K. Huang, C. Xiong, and X. Zhao, Int. J. Mod. Phys. A 29, 50074 (2014).

[50] A. Khmelnitsky and V. Rubakov, J. Cosmol. Astropart. Phys. 2, 19 (2014).

[51] D. J. E. Marsh and J. Silk, Mon. Not. R. Astron. Soc. 437, 2652 (2014).

[52] L. E. Strigari, J. S. Bullock, M. Kaplinghat, A. V. Kravtsov, O. Y. Gnedin, K. Abazajian, and A. A. Klypin, Astrophys. J. 652, 306 (2006).

[53] M. Viel, G. D. Becker, J. S. Bolton, and M. G. Haehnelt, Phys. Rev. D 88, 043502 (2013).

[54] A. Schneider, D. Anderhalden, A. V. Macciò, and J. Diemand, Mon. Not. R. Astron. Soc. 441, L6 (2014).

[55] C. Schultz, J. Oñorbe, K. N. Abazajian, and J. S. Bullock, Mon. Not. R. Astron. Soc. 442, 1597 (2014).

[56] B. Bozek, D. J. E. Marsh, J. Silk, and R. F. G. Wyse, ArXiv e-prints (2014), 1409.3544.

[57] J. P. Gardner, J. C. Mather, M. Clampin, R. Doyon, M. A. Greenhouse, H. B. Hammel, J. B. Hutchings, P. Jakobsen, S. J. Lilly, K. S. Long, et al., Space Sci. Rev. 123, 485 (2006).

[58] E. Calabrese, R. Hložek, N. Battaglia, J. R. Bond, F. de Bernardis, M. J. Devlin, A. Hajian, S. Henderson, J. C. Hil, A. Kosowsky, et al., J. Cosmol. Astropart. Phys. 8, 10 (2014).

[59] H.-Y. Schive, Y.-C. Tsai, and T. Chiueh, Astrophys. J. Suppl. 186, 457 (2010).

[60] E. Seidel and W.-M. Suen, Phys. Rev. D 42, 384 (1990).

[61] L. M. Widrow and N. Kaiser, Astrophys. J. 416, L71 (1993).

[62] F. S. Guzmán and L. A. Ureña-López, Astrophys. J. 645, 814 (2006).

[63] D. S. Reed, R. Bower, C. S. Frenk, A. Jenkins, and T. Theuns, Mon. Not. R. Astron. Soc. 374, 2 (2007).

[64] G. L. Bryan and M. L. Norman, Astrophys. J. 495, 80 (1998).

[65] D. J. Mortlock, S. J. Warren, B. P. Venemans, M. Patel, P. C. Hewett, R. G. McMahon, C. Simpson, T. Theuns, E. A. Gonzáles-Solares, A. Adamson, et al., Nature 474, 616 (2011). 